\title{
Guest Editorial: Visual Analytics in Multimedia-Opportunities and Research Challenges
}

$\mathbf{W}$ ITH the rapid development of Internet and online media platforms such as YouTube, Pandora, and Instagram, multimedia data characterized by high volume, high velocity, and/or high variety have been generated daily. Millions, even billions of videos, music, and photos are being shared and spread on these platforms every day, unleashing a proliferation of opportunities for multimedia data analysis. Traditional research topics in multimedia focus on developing techniques to manage large collections of data (e.g., indexing, multimedia search, and retrieval techniques), or to analyze the multimedia data, for example, to summarize the semantics or detect outliers from images or video streams, or to support the development of various multimedia applications such as VR and AR. To achieve these goals, data mining and machine learning techniques have been extensively and successfully applied in the field of multimedia. However, these approaches usually rely on labeled data for modeling, training, or result validation, thus challenges still remain when these data are noisy, uncertain, or incomplete.

Visual analytics is an emerging research direction that focuses on data exploration and analysis with a seamless integration of interaction, visualization, and analysis. By showing data and analysis results in context, a visualization can display rich evidence that supports or goes against the analysis results, thus helping with data interpretation and result validation. Analysts can annotate or adjust the results via interactive user interfaces and visualizations to supervise the underlying analysis procedure based on, for example, advanced active learning algorithms, thus producing more reliable or precise analysis results gradually. The combination enables human-centric computational intelligence by effectively integrating human knowledge and expertise with powerful computational algorithms through the high-bandwidth visual processing channel and user interaction.

Recent progress on visualization and visual analytics creates new opportunities for understanding and making sense of largescale, dynamic, heterogeneous multimedia data through "analytical reasoning facilitated by interactive visual interfaces." Existing studies of visual analytics have already produced some insightful results and findings, which demonstrates the success and effectiveness of visual analytics for dealing with the complex multimedia data.

This special issue of the IEEE TRANSACTION ON MULTIMEDIA introduces the readership to the latest innovations in visual analytics for multimedia and provides a spotlight on an emerging and promising area of research within the larger multimedia community.
In the special issue, the first ten papers present visualization or interactive visual analysis techniques developed for summarizing, exploring, or analyzing traditional multimedia data, such as videos, images, and music. The first paper is a survey paper which provides a comprehensive overview of the state-of-the-art research on the visual analytics of social media data and place prior studies in a unified and coherent framework [1]. The next four video analysis papers focus on analysis of video content. Heimerl et al. [2] introduced a visual analysis system for analyzing inherent structure and content of movies. Jiang et al. [3] introduced a hierarchy visualization technique for representing video search results in a visualization-friendly form based on advanced analysis algorithms. Ma et al. [4] introduced a geographic map metaphor that represents the content of a video in multiple granularities to support level-of-detail exploration. Renoust et al. [5] tracked faces in a set of new videos and displayed the results in networks to reconstruct a political social network that demonstrates the evidence of "presidentialization" of the media. Liao et al. [6] utilize visualization techniques to help with video annotation based on active learning. Despite video analysis and summarization, the special issue also covers techniques developed for visual summarization of images [7] and music [10] as well as interactive image analysis [8], [9].

The next five papers present techniques for visually analyzing other types of multimedia data. Xia et al. [11] introduced GameFlow which is a narrative visualization designed for exploring NBA basketball games. Baytas et al. [12] introduced PHENOTREE, a visual analyzing tool that supports an interactive phenotyping process of large-scale electronic health cohorts. Liang et al. [13] introduced a visual analytics method to analyze eye-tracking data in a space-time visualization design. Li et al. [14] analyzed the skeletal interactions based on visualization and analysis of active joint graphs. Liang et al. [15] proposed spin contour, a novel data model for representing 3D shapes based on the $2 \mathrm{D}$ point set.

The last paper presented a novel visualization framework that facilitates the understanding of user preferences and optimizes the directory visualization based on content relevancy and utilization of the smartphone screen [16].

This special issue provides readers with a sample of a variety of visual analytics and visualization techniques for multimedia data. Researchers are exploring a wide range of topics that can help bridge visualization and multimedia, in which new communities are emerging. Many challenging problems remain, but we believe that visual analytics and visualization techniques will become more and more important for the future multimedia research. As what the articles in this issue demonstrate, visual analytics and visualization allow analysts to derive actionable, 
meaningful insights from the vast and complex multimedia data resources and help address the challenges that can be difficult to address by traditional analysis methods. Yet this issue also indirectly highlights some of potential problems that need more attention. These include the lack of standardized methods for creating and evaluating visual analytics tools, the exploration and summarization of large-scale multimedia from different sources, and the uncertainty of the visualization results. All these challenges should be explored in the future research.

\author{
N. CAO, Guest Editor \\ Tongji University \\ Shanghai 200092, China
}

Y. Wu, Guest Editor

Zhejiang University

Hangzhou 310058, China

D. GoTZ, Guest Editor

University of North Carolina at Chapel Hill

Chapel Hill, NC 27599 USA

D. KIEM, Guest Editor

University of Konstanz

Konstanz 78457, Germany

Y.-P. TAN, Guest Editor

Nanyang Technological University

Singapore 639798

\section{REFERENCES}

[1] Y. Wu, N. Cao, D. Gotz, Y.-P. Tan, and D. Keim, "A survey on visual analytics of social media data," IEEE Trans. Multimedia, to be published.

[2] F. Heimerl, K. Kurzhals, M. John, P. Kuznecov, and D. Weiskopf, "Visual movie analytics," IEEE Trans. Multimedia, to be published.

[3] Y.-G. Jiang, J. Wang, Q. Wang, and W. Liu, "Hierarchical visualization of video search results for topic-based browsing," IEEE Trans. Multimedia, to be published.

[4] C. Ma, Y.-j. Liu, G. Zhao, and H. Wang, "Visualizing and analyzing video content with interactive scalable map," IEEE Trans. Multimedia, to be published.

[5] B. Renoust and D.-D. Le, "Visual analytics of political networks from face-tracking of news video," IEEE Trans. Multimedia, to be published.

[6] H. Liao, L. Chen, Y. Song, and H. Ming, "Visualization based active learning for video annotation," IEEE Trans. Multimedia, to be published.

[7] Y. Wu et al., "Monet: A system for reliving your memories by theme-based photo storytelling," IEEE Trans. Multimedia, to be published.

[8] D. Koelma and J. Zahálka, "Multimedia pivot tables for multimedia analytics on image collections," IEEE Trans. Multimedia, to be published.

[9] L. Ma et al., "No-reference retargeted image quality assessment based on pairwise rank," IEEE Trans. Multimedia, to be published.

[10] G. Cantareira, F. Paulovich, and L. G. Nonato, "MoshViz: A Detail+Overview approach to visualize music elements," IEEE Trans. Multimedia, to be published.

[11] J. Xia et al., "GameFlow: Narrative visualization of NBA basketball games," IEEE Trans. Multimedia, to be published.

[12] I. Baytas, K. Lin, F. Wang, A. K. Jain, and J. Zhou, "PHENOTREE: Interactive visual analytics for hierarchical phenotyping from large-scale electronic health record," IEEE Trans. Multimedia, to be published.

[13] H. Liang, G. Sun, and R. Liang, "Looking into saliency model via spacetime visualization," IEEE Trans. Multimedia, to be published.

[14] M. Wei, L. Liang, A. Szymczak, W.-M. Pang, and M. Wang, "Spin contour," IEEE Trans. Multimedia, to be published.

[15] M. Li and H. Leung, "Multiview skeletal interaction recognition using active joint interaction graph," IEEE Trans. Multimedia, to be published.

[16] S. Park, M. Cheung, J. She, and J.-L. Lamothe, "Analytics-driven visualization on digital directory via screen-smart device interactions," IEEE Trans. Multimedia, to be published. 